\title{
Piloting respectful maternity care solutions: Research findings from a study in Zambia
}

Breakthrough RESEARCH

Follow this and additional works at: https://knowledgecommons.popcouncil.org/departments_sbsr-rh How does access to this work benefit you? Let us know!

\section{Recommended Citation}

Breakthrough RESEARCH. 2020. "Piloting respectful maternity care solutions: Research findings from a study in Zambia," Programmatic Research Brief. Washington, DC: Population Council. 


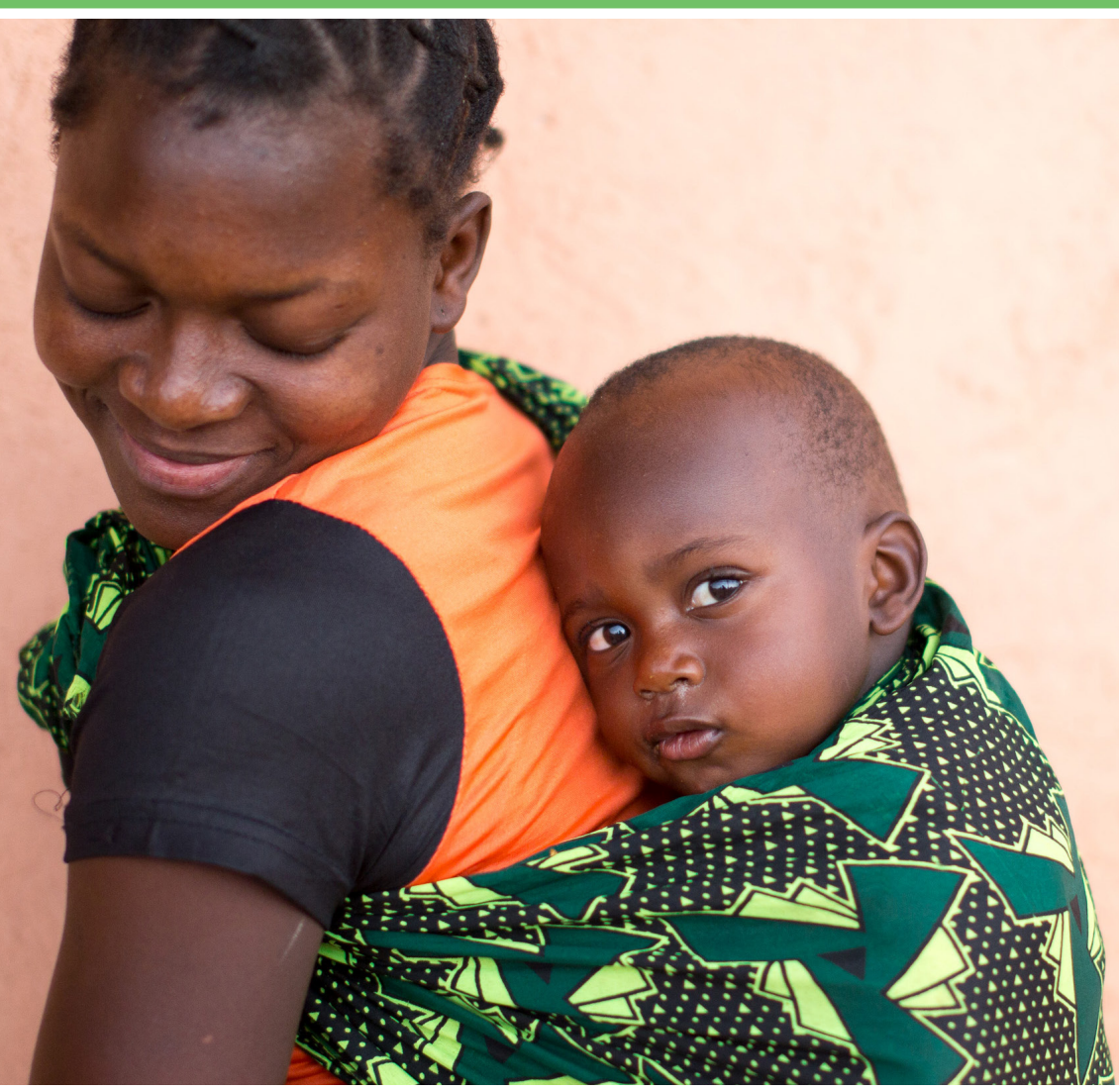

\section{PILOTING RESPECTFUL MATERNITY CARE SOLUTIONS: RESEARCH FINDINGS FROM A STUDY IN ZAMBIA}

This brief details the implementation of a pilot phase of respectful maternity care (RMC) solutions in Chipata, Zambia, under the Breakthrough RESEARCH project. It describes the design of the pilot, the process of implementation by local partner Safe Motherhood $360+(\mathrm{SM} 360+)$, and key learnings from implementation. This is a follow-up to a previous brief that described the formative research findings and solutions developed to promote RMC using behavioral design in the same location.

\section{KEY POINTS}

The solutions, designed through an iterative behavioral design process, were simple for providers to implement, and the tools were easily integrated into existing services by providers during labor and delivery.

Safe Motherhood 360+'s role in engagement of local stakeholders and advocacy for RMC was critical for gaining buy-in at higher levels in the government.

The set of solutions, particularly the BETTER pain management toolkit, shows potential to increase the frequency of encouragement and non-clinical interaction between the provider and the client. 


\section{Background}

RMC, as defined by the World Health Organization, refers to care "organized and provided to all women in a manner that maintains their dignity, privacy and confidentiality, ensures freedom from harm and mistreatment and enables informed choice and continuous support during labor and childbirth (emphasis added)." ${ }^{11}$ Yet a 2019 study completed in four countries found that $35 \%$ of women surveyed had experienced "physical or verbal abuse, or stigma or discrimination" during care-seeking behavior and can dissuade women from having future deliveries in a health facility. ${ }^{3,4}$

In 2018, Breakthrough RESEARCH partner ideas42 conducted qualitative research in close collaboration with the SM360+ project and with the support of USAID/Zambia to identify the behavioral barriers inhibiting providers from consistently providing RMC. Using these research findings, ideas 42 co-created a set of solutions to promote RMC with the Chipata District Health Offices (DHOs), providers, supervisors, and women in labor through an iterative user-testing phase.

The final solutions were the BETTER pain management toolkit, a provider-client promise, and a feedback box (described in Box 1). A summary of the research findings from user testing and descriptions of these solutions can be found in a previous brief. ${ }^{5}$ To support the implementation and improve the effectiveness of these solutions, the team proposed the implementation of two additional solutions: "Fresh Start" facility improvement funds and a values-clarification "Reflection Workshop."

Given the positive results of user testing and encouraging feedback from local stakeholders including the Ministry of Health, DHO, USAID/Zambia, and SM360+, plans were made to conduct a small-scale pilot in 2019.

labor and delivery. ${ }^{2}$ In addition to negatively affecting clinica outcomes, disrespectful maternity care has adverse consequences for women's experience of birth and subsequent

\section{BOX 1 RMC SOLUTIONS}

Below is a complete list of the solutions that comprise the RMC intervention. For more information on the solutions and behavioral barriers they address, please see our previous brief.

BETTER Pain Management Toolkit: BETTER stands for Breathe, Encourage, Turn, Think, and Rub. (1) BETTER Pain Management technique posters; (2) BETTER Pain Management manual; (3) Massage balls; and (4) BETTER Pain Management partograph guide.

Feedback Box: Women were provided with a token upon discharge from the maternity ward and instructed to insert the token into the slot that best reflected the

BFTTER Pain relief helps to promote a fast delivery and healthy mother and baby

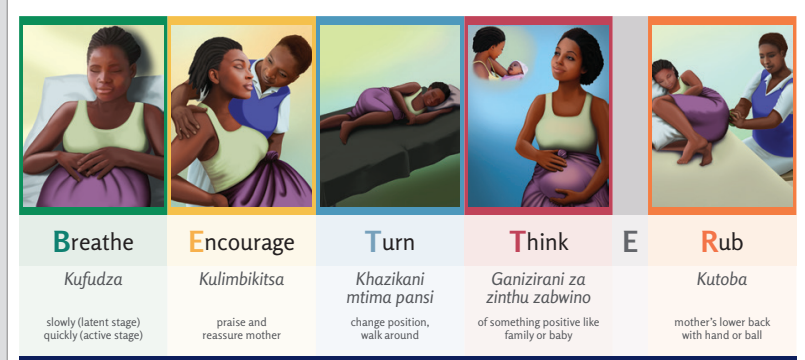

Ask me to do one of these things for you, I am here to help you. Ndifunseni kuti ndichite chimodzi mwa zinthu izi, ndikubwera kudzakuthandizan

BETTER Pain Management technique poster. service they received.

Provider-Client Promise: A promise between provider and patient conducted upon admission to the labor ward.

Fresh Start Funds: Facilities were provided with a small fund, which they used to make small changes to the labor ward to improve the client experience.

Reflection Workshop: A workshop to develop an intention to change care as a facility and introduce solutions. 
This brief describes the design of the pilot, the process of implementing the solutions, key lessons learned from the pilot, and next steps. An upcoming brief will detail the results of the evaluation and provide insights into the effectiveness of the solutions in improving the experience of care for women in labor.

\section{Preparing for Successful Implementation}

\section{Advocating for respectful maternity care}

To prepare for implementation as well as position this work for scale and higher-level impact, our partner SM360+ engaged a range of key stakeholders regarding the importance of RMC. For instance, SM360+ shared this work with the Safe Motherhood and Quality Assurance Technical Working Groups to advocate for RMC in their agendas.

In addition to their own outreach efforts, the Midwives Association of Zambia, which received external funding to develop national guidelines, invited SM360+ to provide input into the guidelines. SM360+ presented the research findings and solution set to this group and reviewed draft national guidelines. As a result, the draft guidelines now include non-clinical approaches (such as encouraging clinicians to use non-pharmacological methods of pain relief such as back rubs, breathing techniques and distraction) to RMC that consider the environment in which providers are implementing.

The Ministry of Health has also been engaged with the program in solution design and implementation, as has the National Directorate of Quality Assurance, which has taken an interest in the RMC approach. To ensure DHO buy-in, the $\mathrm{DHO}$ was involved with all phases of the project, from diagnosing the problem to designing the solution set. The DHO understood the value of promoting RMC and appreciated that the solutions would not disrupt routine service provision; for example, the provider-client promise could be integrated into the existing client admission process. DHO support also enabled the project's access to the health facilities as well as assistance from SM360+ clinical mentors in implementing the solution set. The DHO stayed engaged throughout implementation as SM360+ participated in DHO monthly management meetings in Chipata.

\section{Operationalizing solutions}

A training of trainers ${ }^{\mathrm{a}}$ approach was used to introduce the solutions first among SM360+ clinical mentors who then

aTrainer of trainers approach refers to the strategy of using master trainers to instruct new trainers on a topic or skill. The new trainers are then responsible for conducting future training on the materials.

\section{BOX 2 PILOT DESIGN}

An intervention pilot was conducted to generate lessons learned from implementation of the solutions and to develop insights into the effectiveness of the solutions from October through mid-December 2019.

Five facilities were selected for implementation, including urban and rural health facilities. An additional five facilities were selected as matched facilities to act as comparisons and did not receive the intervention, as part of the evaluation design.

The evaluation methodology was developed collaboratively with SM360+ to ensure that the results of the pilot could be used to enhance future country programming and support efforts to promote RMC more broadly.

conducted the same reflection workshops to train providers; the process for implementation is described on the next page. The reflection workshop for providers sought to affect change as conceptualized in the solution set's theory of change through the following means: (i) generate reflection on the current state of care by providing a space for providers to share their challenges in providing RMC, (ii) drive motivation to change state of care by conducting empathy-building games and discussing the impact of care on client experience, and (iii) solidify commitment to change through individual and joint goal-setting at the facility level. The workshops were designed to be interactive and included role-playing labor scenarios and participatory games using the solutions.

During the reflection workshop, providers were also introduced to the Fresh Start funds, a component of the solution set supported through the project. Each facility was provided with a small fund following the reflection workshop to make changes to the facility and improve client experience in any way that their facility collectively decided. Facility supervisors and providers were meant to align together on the best use of the funds. This solution was meant to empower each facility and its providers to be agents of change in the process of transforming the current state of care. Additionally, given that all facilities differed in management/context, this solution allowed facilities to make decisions about what was most needed in their facility rather than imposing top-down general solutions. Details on how these funds were used and lessons learned are described later in this brief. 


\section{Process of Implementation}

\section{Initial reflection workshop}

The workshop was facilitated by ideas42 in Chipata, Zambia in collaboration with SM360+ staff including clinical mentors. Facility representatives were invited to participate along with the provincial representative from the Midwives Association of Zambia and the provincial health office's Nursing Officer. The workshop took place over the course of two days.

\section{Replication reflection workshops}

Clinical mentors who attended the reflection workshops hosted 1-day replication workshops for clinicians and staff who participate in labor and delivery at each of the five implementing health facilities. Clinical mentors were provided with training materials and facilitator guides as well as the final solutions set, including the BETTER pain management toolkit, feedback box, and posters. If a provider was not present at the replication workshop, the clinical mentor utilized a supplemental guide to orient the provider to the solutions at a later date.

\section{Introduction of Fresh Start funds}

Shortly after the replication workshops, and with support from the clinical mentors, health facilities convened a meeting among supervisors and providers to examine service provision and facility conditions and identify a use for the funds. Facilities were then instructed to use the funds within two weeks.

\section{Ongoing monitoring}

SM360+ clinical mentors provided ongoing support at the health facilities, while supervisors from the DHO provided mentorship and supportive supervisions visits. The clinical mentors included the supervision and monitoring of the designs into their existing responsibilities and visits. In addition, data collectors conducted observation visits to understand how the designs were being used in practice.

\section{Evaluation of pilot}

To generate lessons learned from implementation and potential effectiveness of the solutions, an evaluation of the pilot phase was conducted.

\section{Key Lessons Learned}

Below are takeaways from implementing the solution set, identified through conversations with SM360+ staff facilitating implementation and preliminary themes from interviews with facility staff. As the solutions were not applied at our matched facilities, the lessons below only reflect the experience at the five implementation sites. The next brief will describe full evaluation results once data analysis has been completed.

\section{General reactions from the facility and DHO}

Health facilities have been implementing the solution set since October 2019. Health facility In-Charges, or supervisors of the ward, have appreciated that the designs are simple and easy for providers to implement. The In-Charges also highlighted how each of the solutions complement one another, which has allowed for improvements in the way that providers administer care. For example, the use of the providerclient promise facilitated providers' use of the BETTER pain management tools. As one In-Charge stated:

[The designs] don't work at the same time; they work at different times but they are all helpful.... If I say I remove the feedback box, how are we going to know if the client is happy or not? Maybe the promises made to the client by the provider were not maintained so this can be reflected in the feedback.... If there is no feedback box, how will we know if clients are satisfied or unsatisfied with our services? And if there is no provider-client promise, how will the client know she is supposed to be treated in a respectful way?

One positive unintended outcome was that the designs inspired the facilities to consider other ways they could improve the women's experience of care. For example, at one facility the In-Charge was inspired by the BETTER pain management techniques and suggested hanging posters in

The solutions inspired supervisors to make other changes to improve care for patients beyond the solutions themselves.

the labor ward that illustrated a happy family to help distract women during painful deliveries. Another In-Charge suggested educating the pregnant women during antenatal care on what to anticipate during labor and delivery by having them tour the labor ward.

The DHO has also stated their enthusiasm about the impact the designs could have on improving quality of care and 
is especially interested in how improved client experience may influence care-seeking behavior. They were particularly encouraged by the provider-client promise and its impact on these two areas and have begun thinking about how it could be adopted for scale-up. They also were excited by the feedback box, as it provided clients with a way to voice their satisfaction in an anonymous manner.

\section{Results from reflection and replication workshops}

Using a training of trainers' approach was an effective way to train health providers as it created a safe environment. Providers felt comfortable asking questions and having honest discussions about client interactions given the peer-to-peer nature of the training. Implementing staff from SM360+ noted that the peer-to-peer approach was particularly successful compared with other top-down trainings. Providers also reported that they enjoyed the interactive format of the workshops and commented that the interactive elements allowed them to reflect on the care they provide. Clinical mentors were appreciative that the workshops were held at the facilities rather than having the providers travel off-site because it allowed providers to focus and engage.

Several In-Charges reported that the replication workshops changed how the providers understood their role during labor and delivery. Providers understood that they should be intentional when giving clients instructions, as it could improve client cooperation. Another In-Charge noted that many providers were familiar with some pain management techniques but hadn't been implementing them; the workshop reinforced their importance.

\section{Use of Fresh Start funds}

Across facilities, In-Charges asked providers to think about how they would prioritize the use of Fresh Start funds. Afterward, a meeting was called with the health facility's financial team to vote on the best use of the funds. Table 1 describes how the funds were used at each facility involved in implementation.

\section{TABLE 1 USE OF FRESH START FUNDS BY FACILITY}

\begin{tabular}{|c|c|}
\hline & $\begin{array}{l}\text { Facility \#1 } \\
\text { Determining that women often arrive late to the facility for delivery, providers decided to use their funds } \\
\text { to purchase mattresses for the mothers' waiting shelter to encourage more women to stay there ahead } \\
\text { of their delivery. Since this change, providers report that many more women are coming to wait at the } \\
\text { mothers' shelter before labor begins. The facility also purchased oxytocin with their funds, as there had } \\
\text { been a stock-out at the provincial level. }\end{array}$ \\
\hline & $\begin{array}{l}\text { Facility \#2 } \\
\text { Because the facility did not have enough mattresses for each woman in the postnatal ward, women would } \\
\text { often share a mattress after delivering. To remedy this, the facility purchased additional mattresses. In } \\
\text { addition, they bought a radio that could be used in the postnatal or labor ward to provide a distraction to } \\
\text { women while in labor. The facility also purchased oxytocin due to stock-outs. }\end{array}$ \\
\hline & $\begin{array}{l}\text { Facility \#3 } \\
\text { In order to provide a distraction to women in labor and delivery, the facility purchased a television for the } \\
\text { delivery ward. }\end{array}$ \\
\hline & $\begin{array}{l}\text { Facility \#4 } \\
\text { The facility only had one blood-pressure machine, forcing providers to seek it out before they could use it } \\
\text { during labor and delivery. This facility purchased an additional blood-pressure machine for the labor and } \\
\text { delivery ward. In addition, they purchased a fetal doppler machine. Women in particular were very excited } \\
\text { by the fetal doppler machine, since it allowed them to hear their baby's heartbeat. In addition, because } \\
\text { the facility lacks running water, large buckets were purchased that could be used to store water and help } \\
\text { clean the delivery ward. }\end{array}$ \\
\hline .000 & $\begin{array}{l}\text { Facility \#5 } \\
\text { Facility staff wanted to improve the environment of the labor ward by providing women with more } \\
\text { privacy. The facility purchased curtains and screens to divide the beds in the labor ward and repainted the } \\
\text { labor ward to refresh it for clients. }\end{array}$ \\
\hline
\end{tabular}


The use of the Fresh Start funds demonstrates how each facility envisioned enhancing patient experience in their setting. At the same time, these insights also highlight the challenge in placing more focus on enhancing client experience when there are other clinical demands; for instance, recent stock-outs led facilities to use some of the funding to purchase oxytocin, which, while critical for prevention and management of labor complications, does not contribute to enhancing the non-clinical client experience.

\section{Response to provider-client promise}

Some providers were initially hesitant about the providerclient promise and assumed clients would be reluctant to sign the document. Providers were encouraged by clinical mentors to begin implementation and not assume clients would refuse, given that during user testing, this was not an issue that arose. As implementation began, providers appreciated and were excited by the promise, and recommended continuing the practice after the pilot phase. As a result of signing the agreement, providers said they felt bound to the commitments and that they would reflect on them while providing care.

As planned, women from the Safe Mother Action Groups (SMAG) shared the provider-client promise with women prior to labor and delivery through community outreach and during antenatal care as well as upon admission for delivery. However, in one facility this practice led to some confusion: Providers did not administer the provider-client promise upon admission to the delivery ward, believing it to be unnecessary as women had already received the promise during antenatal care. In other facilities, this challenge did not occur and providers applied the promise consistently during deliveries.

\section{Takeaways from the BETTER pain management toolkit}

Providers reported that the use of the BETTER pain management toolkit increased interactions with the client. Prior to using the pain management toolkit, one In-Charge stated that providers did not spend a lot of time with the clients outside of clinical tasks. However, with the pain management toolkit and the BETTER partograph guide, an In-Charge reported that providers were assisting the client more frequently. One In-Charge remarked that a client had told him that it was like "she wasn't even in labor" with the pain management support she had received.

Providers reported that the BETTER pain management tools were very helpful and appreciated that there were techniques that the client could use on herself or with the support of a SMAG, since at times the providers are very busy. Many providers liked the massage balls, a component of the

\section{One In-Charge remarked that a client} had told him that it was like "she wasn't even in labor" with the pain management support she had received.

BETTER pain management toolkit, and remarked that this was among clients' favorite techniques; one provider mentioned that the ball is "working wonders" for pain management.

\section{Response to the feedback box}

The feedback box was one of the more challenging solutions to implement with fidelity. At initial presentation of the feedback box during implementation, some providers expressed hesitancy as they expected primarily negative feedback. However, providers have reported that clients have been more appreciative of their services than originally envisioned. During data collectors' facility observations, providers

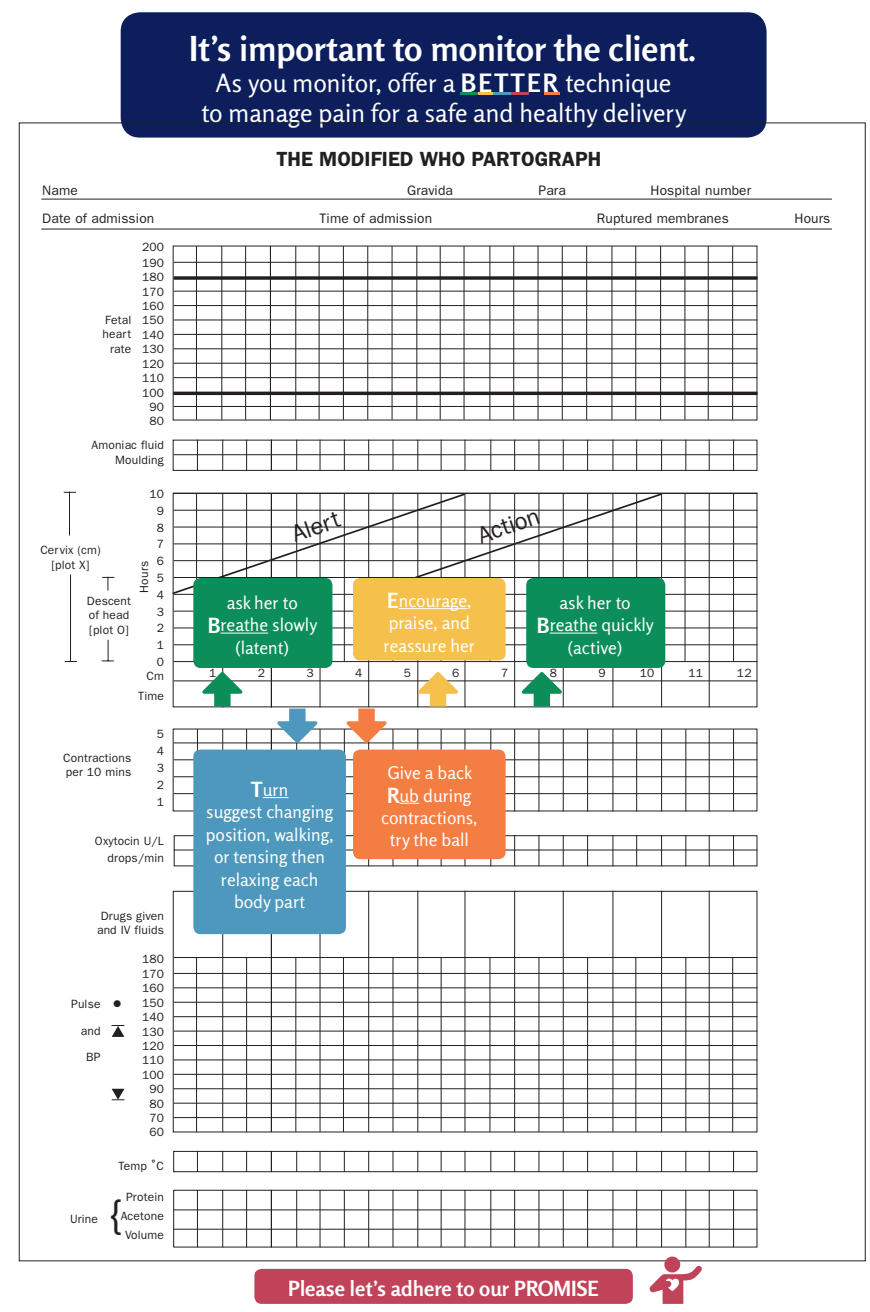

One In-Charge reported that providers were assisting the client more frequently with the use of the pain management toolkit and the BETTER partograph guide (above). 
appeared to give clear and unbiased instructions on how to use the feedback boxes and what each of the three slots represented. Additionally, across most facilities, the feedback was reviewed monthly during regular meetings. There were some challenges around how the feedback box was understood by the community and its general management, which points to opportunities to better leverage the SMAGs in sharing its purpose in the community.

\section{Next Steps}

Lessons learned from the pilot phase suggest the potential of the solutions to promote RMC while also highlighting opportunities for further refinement of the solutions in preparation for scale-up. For instance, pilot results highlight the role that SMAGs and other community actors may play to facilitate understanding of the solutions in the community setting. Allowing facilities latitude to make improvements during the pilot and share these adaptations was a successful strategy in ensuring continued co-creation of the solutions together with the providers and supervisors leading their direct implementation. SM360+'s continued engagement with the DHO was also critical to a successful pilot.

As part of this pilot, baseline and endline data are being collected with both providers and clients to assess the effectiveness of the solutions. These results and additional learnings will be documented in an upcoming brief. The implementation learnings described in this brief, in addition to the quasi-experimental findings of the evaluation which will be featured in the next brief, will help to further refine solutions to increase their feasibility and impact. Upon completion of the evaluation, results will be shared and discussed with the $\mathrm{DHO}$ and Ministry of Health with the support of SM360+. 


\section{REFERENCES}

${ }^{1}$ World Health Organization (WHO) Reproductive Health Library. 2018. "WHO recommendation on respectful maternity care during labour and children." Geneva: WHO. Available from:

https://extranet.who.int/rhl/topics/preconception-pregnancy-childbirth-and-postpartum-care/ care-during-childbirth/who-recommendation-respectful-maternity-care-during-labour-and-childbirth

${ }^{2}$ Bohren, M. A. et al. 2019. "How women are treated during facility-based childbirth in four countries: a cross-sectional study with labour observations and community-based surveys," The Lancet 394(10210): 1750-1763. doi: 10.1016/S0140-6736(19)31992-0

${ }^{3}$ Kujawski, S. et al. 2015. "Association between disrespect and abuse during childbirth and women's confidence in health facilities in Tanzania," Maternal and Child Health Journal 19(10): 2243-2250. doi: 10.1007/ s10995-015-1743-9

${ }^{4}$ Faye, A., M. Niane, \& I. Ba. 2011. "Home birth in women who have given birth at least once in a health facility: contributory factors in a developing country." Acta Obstetricia et Gynecologica Scandinavica 90(11): 123943. doi: 10.1111/j.1600-0412.2011.01163.x

${ }^{5}$ Breakthrough RESEARCH. 2019. "Enhancing respectful maternal care during labor and delivery," Programmatic Research Brief. Washington, DC: Population Council. https://breakthroughactionandresearch.org/wp-content/uploads/2019/02/Respectful-Care-Brief.pdf

\section{Acknowledgments}

This programmatic research brief describes work led by ideas 42 under Breakthrough RESEARCH. This brief and the work it describes is possible through the work and support of SafeMotherhood360+, Population Council Zambia, and Population Council, Washington, DC.

\section{Suggested citation:}

Breakthrough RESEARCH. 2020. "Piloting respectful maternity care solutions: research findings from a study in Zambia," Programmatic Research Brief. Washington DC: Population Council.

\section{Front cover photo credit:}

(C2015 Bread for the World (CC BY-ND 2.0/No modifications)

(C) 2020 The Population Council. All rights reserved.
Email

BreakthroughResearch@popcouncil.org

\section{Breakthrough RESEARCH | Population Council}

4301 Connecticut Ave., NW, Suite 280 | Washington, DC 20008

+12022379400 | breakthroughactionandresearch.org

\section{(P) USAID}

Breakthrough RESEARCH is made possible by the generous support of the American people through the United States Agency for International Development (USAID) under the terms of cooperative agreement no. AIDOAA-A-17-00018. The contents of this document are the sole responsibility of Breakthrough RESEARCH and Population Council and do not necessarily reflect the views of USAID or the United States Government.
POPULATION COUNCIL

Ideas. Evidence. Impact.

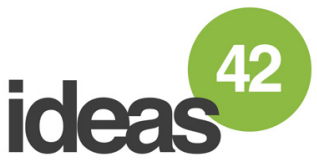

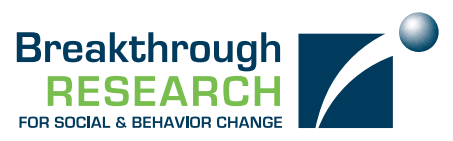

Our project turns evidence into action by providing thought provoking guidance to improve SBC policy and programming, with the goal of improving the cost-effectiveness of USAID's health and development strategies. Breakthrough RESEARCH catalyzes SBC by conducting state-of-the-art research and evaluation and promoting evidence-based solutions to improve health and development programs around the world. Breakthrough RESEARCH is a consortium led by the Population Council in partnership with Avenir Health, ideas42, Institute for Reproductive Health at Georgetown University, Population Reference Bureau, and Tulane University. 